\title{
More Compactification for Differential Systems
}

\author{
Harry Gingold, Daniel Solomon \\ Department of Mathematics, West Virginia University, Morgantown, USA \\ Email: gingold@math.wvu.edu, solomon@math.wvu.edu
}

Received October 29, 2012; revised November 30, 2012; accepted December 8, 2012

\begin{abstract}
This article is a review and promotion of the study of solutions of differential equations in the "neighborhood of infinity" via a non traditional compactification. We define and compute critical points at infinity of polynomial autonomuos differential systems and develop an explicit formula for the leading asymptotic term of diverging solutions to critical points at infinity. Applications to problems of completeness and incompleteness (the existence and nonexistence respectively of global solutions) of dynamical systems are provided. In particular a quadratic competing species model and the Lorentz equations are being used as arenas where our technique is applied. The study is also relevant to the Painlevé property and to questions of integrability of dynamical systems.
\end{abstract}

Keywords: Nonlinear; Polynomial; Compactification; Ultra Extended Euclidean Space; Critical Point; Equilibrium Point; Critical Point at Infinity; Critical Direction at Infinity; Basin of Divergence; Basin of Convergence; Ideal Solutions; Asymptotic; Stability; Global; Globally Asymptotically Stable; Jacobian; Painleve Analysis, Competing Species; Model; Lorenz Equations; Periodic Surface; Differential Geometry; Attractor; Repeller

\section{Introduction}

The projection of the real line on a circle is a form of compactification that was known to Greek mathematiccians before the commeon era. In 1881 Poincaré [1], studied limit cycles "at infinity" of two dimensional polynomial differential equations via compactification. Although the paper contained errors that were addressed more than a hundred years later by Roeder [2], the originnal ideas had lasting impact. An early study of differential equations via compactification was carried out by Bendixson [3]; see Andronov et al. ([4], p. 216). Bendixson used the stereographic projection that does not account for all directions at infinity. See e.g. Ahlfors [5] and Hille [6], for versions of the stereographic projection. The Poincaré compactification is adopted in various textbooks on differential equations. See also [7-10]. It is widely used to study critical points at infinity. Compare e.g. with the studies of Chicone and Sotomayor [11], Cima and Llibre [12], Schlomiuk and Vulpe [13], and their references. It is noteworthy that the stereographic projection is obtained by Y. Gingold and H. Gingold [14] as a degenerate limit of a family of compactifications that account for all directions at infinity. However, that compactification is akin to the Poincaré compactification [1], and possesses radicals that prevent it being a tool for rational approximations. Compactification is an excellent mean to obtain global phase portraits of vector fields of dynamical systems, that include the neigborhood of infinity. [12] is a welcome global analysis and supplement to the analysis of Chen, Guang Qing and Liang, Zhao Jun [15].

This article is dedicated to the review to the exposition and to the promotion of the study of solutions of differential systems and dynamical systems in the "neighborhood of infinity". This study promotes and utilizes a certain non traditional compactification. The treatment is based on a series of papers published in the span of the years 2004 to 2012. We describe the theoretical background necessary to define neighborhoods and critical points at infinity of solutions of differential systems. We develop an explicit formula for the leading asymptotic term of diverging solutions to critical points at infinity. Applications to problems of completeness and incompleteness of dynamical systems are also brought to the fore. In particular quadratic systems and the Lorentz equations are being used as examples where new and old results are obtained. The quadratic system of competing species is utilized as an example of an incomplete system to which a main result, Theorem 14, applies. Theorem 14 is also related to the Painleve property and consequently is related to issues of integrability. The Lorenz system is used as a prototype of a nonlinear quadratic system that is complete for a much larger set of parameters known before. A simple bijection, $y=\left(1-x^{\dagger} x\right)^{-1} x, x, y \in \mathbb{R}^{k}$, that has a rich geometrical interpretation, plays a major 
role in our study.

Details are provided when new results are derived or an important point of view is stressed. Otherwise, we defer for details to the original papers. Traditionally, various aspects of an article and comparisons with references are provided in an introduction section. However, we prefer to postpone such comparisons and comments to subsequent sections with the hope that the delay will make the ideas more tangible. We are happy to acknowledge the influence of the article by Elias and Gingold [14] on the application of compactification methods to the study of differential systems of equations.

We could not find in the text books on discrete dynamical systems the utilization of compactification techniques. A modest attempt to fill up this large gap is given in H. Gingold [16-18].

The order of presentation in this article runs as follows.

In Section 2 we define what divergence to infinity means in $\mathbb{R}^{k}$ and we extend $\mathbb{R}^{k}$ to a larger set to be called the Ultra Extended $\mathbb{R}^{k}$.

In Section 3 we discuss properties of the compactification $y=\left(1-x^{\dagger} x\right)^{-1} x$, some of its geometrical interpretations and the metric induced by it in the Ultra Extended $\mathbb{R}^{k}$.

In Section 4 we discuss the new equations resulting from the transformation of an initial value problems of a polynomial differential system $y^{\prime}=f(y)$ under the compactification $y=\left(1-x^{\dagger} x\right)^{-1} x$.

In Section 5 we define what a critical point $\infty p$ of $y^{\prime}=f(y)$ is, and we prove a theorem that a polynomial differential system has at least one critical point in the Ultra Extended $\mathbb{R}^{k}$.

In Section 6 we derive an explicit formula for a Jacobian associated with a critical point $\infty p$ of $y^{\prime}=f(y)$. We obtain an explicit leading asymptotic term of solutions of $y^{\prime}=f(y)$ that diverge to $\infty p$. From this explicit formula old and new results follow.

In Section 7 we discuss the ramification of the previous sections on a large family of quadratic systems.

In Section 8 we show how the compactification techniques shed new light on the completeness of the celebrated Lorenz system. We also identify an extension of the attractor. The utility of ideal solutions $y(t) \equiv \infty$ is brought to the fore.

In Section 9 we study fields of Lorentz like systems near infinity.

\section{Divergence in the Ultra Extended $\mathbb{R}^{k}$}

Denote by $y$ a column vector in $\mathbb{R}^{k}$. Let $y^{\dagger}=\left(y_{1}, y_{2}, \cdots, y_{k}\right)$ denote a row vector that is the transpose of $y$. In particular let $\hat{0}^{\dagger}=(0, \cdots, 0)$ be the transpose of the zero vector. Let $f^{\dagger}(y):=\left(f(y)_{1}, f(y)_{2}, \cdots, f(y)_{k}\right)$ be a vector field in $\mathbb{R}^{k}$ where $f(y)_{j}, j=1,2, \cdots, k$ are scalar polynomial functions. Denote by $\left(t_{\min }, t_{\max }\right)$ the maximal interval of existence of a solution of a differential equation $y^{\prime}=\frac{\mathrm{d} y}{\mathrm{~d} t}=f(y)$. We say that $\frac{\mathrm{d} y}{\mathrm{~d} t}=f(y)$ is a polynomial differential system of degree $L$ if the vector function $f(y)$ is given by

$$
f(y)=f_{0}(y)+f_{1}(y)+\cdots+f_{L-1}(y)+f_{L}(y),
$$

where $f_{j}(y), j=0,1,2, \cdots, L$ are homogeneous polynomials column vectors of degree $j$ and $f_{L}(y) \neq \mathbf{0}$ for some $y \in \mathbb{R}^{k}$. [Note the difference between $f(y)_{j}$ and $\left.f_{j}(y)\right]$.

What does it mean that a sequence of points $z(n) \in \mathbb{R}^{k}$, $n=1,2, \cdots$ converges to infinity in $\mathbb{R}^{k}$ ? What does it mean that a continuous vector function $y(t) \in C\left(t_{\min }, t_{\max }\right)$ diverges in the direction $p$ to infinity in $\mathbb{R}^{k}$. There are at least two different definitions.

Definition 1. We say that the sequence $z(n) \in \mathbb{R}^{k}$, $n=0,1,2, \cdots$ diverges to infinity if

$$
\lim _{n \rightarrow \infty} z(n)^{\dagger} z(n)=+\infty \text {. }
$$

However, This definition is too restrictive for various purposes; e.g., mathematical physics. It blurs the distinction between the different directions at infinity. A definition that distinguishes between all directions at infinity requires the following.

Definition 2. We say that the unbounded sequence $z(n), n=0,1,2, \cdots$ diverges in the direction $p$ to infinity or diverges to $\infty p, p^{\dagger} p=1$, if (2) holds and

$$
\lim _{n \rightarrow \infty}{\sqrt{z(n)^{\dagger} z(n)}}^{-1} z(n)=p .
$$
by

The continuous analog of the definition above is given

Definition 3. We say that the vector function

$y(t) \in C\left(t_{\min }, t_{\max }\right)$ diverges in the direction $p$ to infinity, or diverges to $\infty p$ and we denote

$$
\lim _{t \rightarrow t_{\min }^{+}} y(t)=\infty p, \text { or } \lim _{t \rightarrow t_{\max }^{-}} y(t)=\infty p,
$$

if we have

$$
\begin{aligned}
& \lim _{t \rightarrow t_{\text {max }}^{-}} y^{\dagger}(t) y(t)=\infty \text {, and } \\
& \lim _{t \rightarrow t_{\max }^{-}} x(t)=\lim _{t \rightarrow t_{\text {max }}^{-}}{\sqrt{y^{\dagger}(t) y(t)}}^{-1} y(t)=p,
\end{aligned}
$$

or

$$
\begin{aligned}
& \lim _{t \rightarrow t_{\min }^{+}} y^{\dagger}(t) y(t)=\infty \text {, and } \\
& \lim _{t \rightarrow t_{\min }^{+}} x(t)=\lim _{t \rightarrow t_{\min }^{+}}{\sqrt{y^{\dagger}(t) y(t)}}^{-1} y(t)=p .
\end{aligned}
$$

Definition 1 is compatible with a common one in complex analysis that is associated with the extended 
complex plane. Just one geometrical point corresponding to the symbol $\infty$ augments $\mathbb{R}^{2}$. It is compatible with the compactification that is given by the stereographic projection. See e.g. [5,6].

Definition 4. Denote by $E \mathbb{R}^{k}$ the union of $\mathbb{R}^{k}$ and an ideal point IP and call it the extended $\mathbb{R}^{k}$ where

$$
I P:=\{\infty\}, E \mathbb{R}^{k}:=\mathbb{R}^{k} \cup I P .
$$

However, compatible with Definition 3 we need a larger ideal set ID. Analogous to Y. Gingold and $\mathrm{H}$. Gingold [14], we define below an Ultra Extended $\mathbb{R}^{k}$ and produce an induced metric in Section 3.

Definition 5. Denote by $U E \mathbb{R}^{k}$ the union of $\mathbb{R}^{k}$ and a certain ideal set ID and call it the Ultra extended $\mathbb{R}^{k}$ where

$$
I D:=\left\{\infty p \mid p^{\dagger} p=1, p \in \mathbb{R}^{k}\right\}, U E \mathbb{R}^{k}:=\mathbb{R}^{k} \cup I D .
$$

As seen in the sequel there is good reason to introduce nonlinear transformations that will allow us to reduce the investigation of differential systems with unbounded solutions to the investigation of differential systems with a-priori bounded solutions.

\section{Compactification and a Metric}

In preparation to transforming the equation $y^{\prime}=f(y)$ we need a diffeomorphism that will facilitate computations and will take the space $\mathbb{U} \mathbb{R}^{k}$ into a bounded set. We sketch the main ideas. For more details see H. Gingold [19]. We project the point $y=\left(y_{1}, \cdots, y_{k}, 0\right) \in \mathbb{R}^{k+1}$ through the point $(0, \cdots, 0,1)$ on the surface (8)

$$
x_{k+1}=\left(x_{1}^{2}+\cdots+x_{k}^{2}\right)^{1 / 2}
$$

and single out $Z=\left(x_{1}, x_{2}, \cdots, x_{k}, x_{k+1}\right)$ as one of the two points of intersection of the parabolic surface (8) and the straight line connecting $\left(y_{1}, \cdots, y_{k}, 0\right)$ and $(0, \cdots, 0,1)$. The determination of $Z$ will be done by the determination of a certain branch of a multi valued function as given below. Then, all the points $y=\left(y_{1}, \cdots, y_{k}, 0\right)$ map onto a parabolicbowl with coordinate $x_{k+1}=\left(x_{1}^{2}+\cdots+x_{k}^{2}\right)^{1 / 2}<1$, $\left(x_{1}, \cdots, x_{k}, x_{k+1}\right) \in \mathbb{R}^{k+1}$ and all the points $\infty p, p^{\dagger} p=1$, map onto the "circle" with $x_{k+1}=\left(x_{1}^{2}+\cdots+x_{k}^{2}\right)^{1 / 2}=1$. Denote by $U$ the unit ball and by $\partial U$ its boundary.

$$
\begin{aligned}
& U:=\left\{x \in \mathbb{R}^{2} \mid x^{\dagger} x \leq 1\right\}, \\
& \partial U:=\left\{x \in \mathbb{R}^{2} \mid x^{\dagger} x=1\right\} .
\end{aligned}
$$

Denote

$$
r=\sqrt{y^{\dagger} y}=\|y\|,\|x\|:=\sqrt{x^{\dagger} x}=R .
$$

The transformation

$$
y=\left(1-R^{2}\right)^{-1} x \Rightarrow r=\frac{R}{1-R^{2}}
$$

is shown in [19] to be a bijection from $\mathbb{R}^{k}$ onto the interior of $U$. It is also a bijection from the ideal set $I D:=\left\{\infty p \mid p^{\dagger} p=1\right\}$ onto $\partial U$. The inverse of $y=\frac{x}{1-x^{\dagger} x}$ in $R<1$ is defined by the branch

$$
x=\frac{2 y}{1+\sqrt{1+4 y^{\dagger} y}}, R=\frac{2 r}{1+\sqrt{1+4 r^{2}}} .
$$

The compactification (12) induces a metric in $U E \mathbb{R}^{k}$ in a natural manner. We consider two points $y, \hat{y} \in U E \mathbb{R}^{k}$. Denote their images under the above bijection by $Z, \hat{Z}$ respectively. Let \|\| denote the Euclidean norm. Define a positive definite function $M(y, \hat{y})$ by

$$
M(y, \hat{y}):=\|Z-\hat{Z}\| \text {. }
$$

Put,

$$
\begin{gathered}
y^{\dagger}=\left(y_{1}, \cdots, y_{k}\right) \text { or } y=\infty p, p^{\dagger} p=1, \\
\hat{y}^{\dagger}=\left(\hat{y}_{1}, \cdots, \hat{y}_{k}\right) \text { or } \hat{y}=\infty \hat{p}, \hat{p}^{\dagger} \hat{p}=1 \\
\hat{r}=\sqrt{\hat{y}^{\dagger} \hat{y}}=\|\hat{y}\|, \theta=\frac{2}{1+\sqrt{1+4 r^{2}}}, \hat{\theta}=\frac{2}{1+\sqrt{1+4 \hat{r}^{2}}},
\end{gathered}
$$

The next theorem borrowed from H. Gingold [19] shows how to make the $U E \mathbb{R}^{k}$ a complete metric space. Then, divergence of solutions of dynamical systems is dealt with by convergence in the induced metric.

Theorem 6. The Ultra Extended $\mathbb{R}^{k}$ is a complete metric space with respect to the chordal metric $M(y, \hat{y}):=\|Z-\hat{Z}\|$. It is given by the following.

$$
\begin{gathered}
M(y, \hat{y})=\sqrt{\theta \hat{\theta}\left\{\|y-\hat{y}\|^{2}-\frac{(\theta-\hat{\theta})^{2}(1-\theta \hat{\theta})}{(\theta \hat{\theta})^{2}}\right\}} \text { if } y, \hat{y} \in \mathbb{R}^{k}, \\
M(y, \hat{y})=\sqrt{\left(1-2 p^{\dagger} \hat{\theta} \hat{y}+\hat{\theta}^{2} \hat{r}^{2}\right)+\left(1-\hat{\theta}^{2} \hat{r}^{2}\right)^{2}} \\
\text { if } y=\infty p, p^{\dagger} p=1, \hat{y} \in \mathbb{R}^{k}, \\
M(y, \hat{y})=\sqrt{2\left(1-p^{\dagger} \hat{p}\right)} \\
\text { if } y=\infty p, p^{\dagger} p=1, \hat{y}=\infty \hat{p}, \hat{p}^{\dagger} \hat{p}=1,
\end{gathered}
$$

Proof. See [19] for details.

See e.g. Willard [20] for topics of compactification in general topolgy.

We turn now to a set of new differential equations resulting from compactifying the differential system $\frac{\mathrm{d} y}{\mathrm{~d} t}=f(y)$.

\section{Compactifyng a Polynomial Differential System}

Put 


$$
\begin{aligned}
\tilde{f}= & \tilde{f}\left(x,\left(1-R^{2}\right)\right):=\left(1-R^{2}\right)^{L} f\left(\left(1-R^{2}\right)^{-1} x\right) \\
= & \left(1-R^{2}\right)^{L} f_{0}(x)+\left(1-R^{2}\right)^{L-1} f_{1}(x)+\cdots \\
& +\left(1-R^{2}\right)^{2} f_{L-2}(x)+\left(1-R^{2}\right)^{1} f_{L-1}(x)+f_{L}(x) .
\end{aligned}
$$

Then the following proposition holds.

Proposition 7. The compactification (11) takes the differential system $y^{\prime}=f(y)$ into the differential system

$$
\frac{\mathrm{d} x}{\mathrm{~d} t}=\frac{\left[\left(1+R^{2}\right) I-2 x x^{\dagger}\right] \tilde{f}\left(x,\left(1-R^{2}\right)\right)}{\left(1+R^{2}\right)\left(1-R^{2}\right)^{L-1}},
$$

with

$$
\frac{\mathrm{d}\left(1-R^{2}\right)}{\mathrm{d} t}=-\frac{2\left(x^{\dagger} \tilde{f}\left(x,\left(1-R^{2}\right)\right)\right)}{\left(1+R^{2}\right)\left(1-R^{2}\right)^{L-2}} .
$$

Moreover, consider $x$ and $t$ as functions of a new independent variable $\tau \in(-\infty, \infty)$ where

$$
\begin{aligned}
& \frac{\mathrm{d} x}{\mathrm{~d} \tau}=\left(1+R^{2}\right) \tilde{f}\left(x,\left(1-R^{2}\right)\right)-2\left(x^{\dagger} \tilde{f}\left(x,\left(1-R^{2}\right)\right)\right) x, \\
& x(0)=x_{0}, \\
& \quad \frac{\mathrm{d} t}{\mathrm{~d} \tau}=\left(1+R^{2}\right)\left(1-R^{2}\right)^{L-1}, t(0)=t_{0} \in\left(t_{\min }, t_{\max }\right), \\
& \quad \frac{\mathrm{d}\left(1-R^{2}\right)}{\mathrm{d} \tau}=-2\left(x^{\dagger} \tilde{f}\left(x,\left(1-R^{2}\right)\right)\right)\left(1-R^{2}\right), \\
& \left(1-R^{2}(0)\right)=1-x_{0}^{\dagger} x_{0} .
\end{aligned}
$$

If $x_{0}^{\dagger} x_{0}<1$ then the initial value problems (17)-(19), possess unique solutions on $-\infty<\tau<\infty$ such that $R^{2}(\tau)=x^{\dagger}(\tau) x(\tau)<1$.

Furthermore the Equation (18) generates a one to one mapping between the variable $\tau$ on $-\infty<\tau<\infty$ and the variable $t$ on $\left(t_{\min }, t_{\max }\right)$. We have then,

$$
\begin{gathered}
t-t_{0}=\int_{0}^{\tau}\left(1+R^{2}\right)\left(1-R^{2}\right)^{L-1} \mathrm{~d} \tau, \\
t_{\text {max }}-t_{0}=\int_{0}^{\infty}\left(1+R^{2}\right)\left(1-R^{2}\right)^{L-1} \mathrm{~d} \tau, \\
t_{0}-t_{\min }=\int_{-\infty}^{0}\left(1+R^{2}\right)\left(1-R^{2}\right)^{L-1} \mathrm{~d} \tau .
\end{gathered}
$$

Furthermore, if $x_{0}^{\dagger} x_{0}=1$, then the initial value problems (17)-(19), possess unique solutions on $-\infty<\tau<\infty$ such that $x^{\dagger}(\tau) x(\tau) \equiv 1$, such that $(1-R(\tau))^{2} \equiv 0$ and such that $t(\tau) \equiv t_{0}$.

Proof. The proof is left as an exercise. Compare with derivations in Elias and Gingold and Gingold and Solomon $[21,22]$.

Also note:

Remark 8. The compactified equations above, contain useful information that will become apparent in the se- quel. The formulas (21), contains the following qualitative information. The larger $L$ the smaller $\left(1-R^{2}\right)^{L-1}$ could become and therefore the smaller $t_{\max }-t_{0}, t_{0}-t_{\min }$ may become.

\section{Critical Points of the Compactified Equation}

The purpose of this section is to discuss a rigorous footing to the notion of a critical point $\infty p$ of a dynamical system using the proposed compactification. If

$$
\lim _{y \rightarrow \infty p} f(y)=\infty q
$$

holds, then $\infty p$ could be a candidate for a critical point of $y^{\prime}=f(y)$ at infinity. Thanks to the definitions and the compactification above, we declare $\infty p$ to be a critical point of $y^{\prime}=f(y)$ if $p$ is a critical point of (17) as follows.

Definition 9. We say that $\infty p$ is a critical (equilibrium) point of $y^{\prime}=\frac{\mathrm{d} y}{\mathrm{~d} t}=f(y)$ at infinity, or that $p$ is a critical direction of $y^{\prime}=f(y)$ at infinity, if there exist a unit vector $p$ such that

$$
\left(p^{\dagger} f_{L}(p)\right) p=f_{L}(p)
$$

If in addition we have $\left[p^{\dagger} f_{2}(p)\right] \neq 0$ then

$$
p=\frac{f_{L}(p)}{p^{\dagger} f_{L}(p)}=\frac{f_{L}(p)}{ \pm \sqrt{\left[f_{L}^{\dagger}(p) f_{L}(p)\right]}}
$$

and we call $\infty p$ a generic critical point at infinity and we call $p$ a generic direction at infinity.

The set of initial points $y_{0} \in \mathbb{R}^{k}$ such that the unbounded solutions of the initial value problem $y^{\prime}=f(y)$, $y\left(t_{0}\right)=y_{0}$, satisfies (4) or (5), is called the basin of divergence of $\infty p$ or the basin of divergence in the $p$ direction. Notice that by this definition at least one value of $x_{0} \neq p$ must be included in the basin of convergence of $p$.

This is a natural definition for a critical point at infinity because of

Proposition 10. If (4) or (5) holds then $p$ must be a finite critical point of (17).

Proof. Notice the identity

$$
\begin{aligned}
& {\left[\left(1+R^{2}\right) I-2 x x^{\dagger}\right] \tilde{f}\left(x,\left(1-R^{2}\right)\right)} \\
& =\left(1+R^{2}\right) \tilde{f}\left(x,\left(1-R^{2}\right)\right)-2\left(x^{\dagger} \tilde{f}\left(x,\left(1-R^{2}\right)\right)\right) x .
\end{aligned}
$$

By virtue of (24), the relation $\tilde{f}(p, 0)-\left(p^{\dagger} \tilde{f}(p, 0)\right) p=\mathbf{0}$ then implies the nonlinear eigenvalue problem (23).

Remark 11. Let $\mu=p^{\dagger} f_{L}(p)<0$ then

$$
\lim _{t \rightarrow t_{\text {max }}^{-}} x(t)=\lim _{t \rightarrow t_{\text {max }}^{-}}{\sqrt{y^{\dagger}(t) y(t)}}^{-1} y(t)=p
$$


is impossible. Let $\mu=p^{\dagger} f_{L}(p)>0$ then

$$
\lim _{t \rightarrow t_{\min }^{+}} x(t)=\lim _{t \rightarrow t_{\min }^{+}}{\sqrt{y^{\dagger}(t) y(t)}}^{-1} y(t)=p
$$

is impossible. Hence, for (4) to hold we must have $p^{\dagger} f_{L}(p) \geq 0$ in forward time and we also must have $p^{\dagger} f_{L}(p) \leq 0$ in order for (5) to hold in backward time.

Also notice:

Remark 12. The set of critical points at infinity of a compactified and parametrized equation are not well defined without a certain normalization that needs to be introduced or is implicitly assumed. In the above treatment we "naturally" but arbitrarily defined a parametrization (17). This determination causes the remaining Equations (17) and (19) to be uniquely determined. However, one may introduce spurious critical points as follows. Consider

$$
\begin{aligned}
\frac{\mathrm{d} x}{\mathrm{~d} s}= & g(x)\left[\left(1+R^{2}\right) \tilde{f}\left(x,\left(1-R^{2}\right)\right)\right. \\
& \left.-2\left(x^{\dagger} \tilde{f}\left(x,\left(1-R^{2}\right)\right)\right) x\right]
\end{aligned}
$$

with

$$
g(x)=\prod_{j=1}^{k}\left(x_{j}-x_{j, 0}\right)^{2 m_{j}}, m_{j} \in \mathbb{N}, \sum_{j=1}^{k} x_{j, 0}^{2}=1 .
$$

Then the equation pertaining to $t$ would be

$$
\frac{\mathrm{d} t}{\mathrm{~d} s}=g(x)\left(1+R^{2}\right)\left(1-R^{2}\right)^{L-1} .
$$

The point $\left(x_{1,0}, x_{2,0}, \cdots, x_{k, 0}\right)$ can be made to be a spurious critical point at infinity. It is noteworthy that the case $k=1$ differs from the case $k>1$. For $k=1$ we have (17) become

$$
\begin{aligned}
\frac{\mathrm{d} x}{\mathrm{~d} \tau} & =\left[\left(1+R^{2}\right) I-2 x x^{\dagger}\right] \tilde{f}\left(x,\left(1-R^{2}\right)\right) \\
& =2\left(1-R^{2}\right) \tilde{f}\left(x,\left(1-R^{2}\right)\right) .
\end{aligned}
$$

Then, $x=( \pm 1)$ are the only two critical points of (17) so that $\infty( \pm 1)$ are the only two critical points at infinity of a scalar polynomial differential equation. However, it seems desirable to choose for $k=1$ a different parametrization with

$$
\begin{gathered}
g(x)=\left(1-R^{2}\right)^{-1}, \frac{\mathrm{d} x}{\mathrm{~d} s}=2 \tilde{f}\left(x,\left(1-R^{2}\right)\right), \\
\frac{\mathrm{d} t}{\mathrm{~d} s}=\left(1+R^{2}\right)\left(1-R^{2}\right)^{L-2} .
\end{gathered}
$$

This will eliminate the common factor of the right hand sides of

$$
\frac{\mathrm{d} x}{\mathrm{~d} \tau}=2 \tilde{f}\left(x,\left(1-R^{2}\right)\right)\left(1-R^{2}\right)
$$

and

$$
\frac{\mathrm{d} t}{\mathrm{~d} \tau}=\left(1+R^{2}\right)\left(1-R^{2}\right)^{L-1} .
$$

Then, if $L>0, x=( \pm 1)$ will not be critical points of $\frac{\mathrm{d} x}{\mathrm{~d} s}=2 \tilde{f}\left(x,\left(1-R^{2}\right)\right)$.

Must every polynomial differential system possess at least one critical point in the Ultra Extended $\mathbb{R}^{k}$ ? The positive answer is given in:

Proposition 13. A polynomial differential system with $L>0$ possesses at least one critical point in the $U E \mathbb{R}^{k}$.

Proof. If $f(y)=\hat{0}$ for some $y \in \mathbb{R}^{k}$ then we are done. Assume now without loss of generality that there does not exist $y \in \mathbb{R}^{k}$ such that $f(y)=\hat{0}$. Consider the relation

$$
\begin{aligned}
\tilde{f}\left(x,\left(1-R^{2}\right)\right) & =\left(1-R^{2}\right)^{L} f\left(\left(1-R^{2}\right)^{-1} x\right) \\
& =\left(1-R^{2}\right)^{L} f(y) .
\end{aligned}
$$

It implies that for $\left(1-R^{2}\right) \neq 0$ also $\tilde{f}\left(x,\left(1-R^{2}\right)\right) \neq 0$. Two possibilities may occur. Either for some $x$ such that $\left(1-R^{2}\right)=0$ we have that $\tilde{f}\left(x,\left(1-R^{2}\right)\right)=0$ then the right hand side of

$$
\frac{\mathrm{d} x}{\mathrm{~d} \tau}=\left[\left(1+R^{2}\right) I-2 x x^{\dagger}\right] \tilde{f}\left(x,\left(1-R^{2}\right)\right)
$$

vanishes and the result follows. If $\tilde{f}\left(x,\left(1-R^{2}\right)\right) \neq 0$ for $x \in U$ then the mapping

$$
w(x):={\sqrt{\tilde{f}\left(x,\left(1-R^{2}\right)\right)^{\dagger} \tilde{f}\left(x,\left(1-R^{2}\right)\right)}}^{-1} \tilde{f}\left(x,\left(1-R^{2}\right)\right)
$$

is a continuous mapping from $U$ into $U$. By Brower's fixed point theorem there exists $x \in U$ such that

$$
w(x)={\sqrt{\tilde{f}\left(x,\left(1-R^{2}\right)\right)^{\dagger} \tilde{f}\left(x,\left(1-R^{2}\right)\right)}}^{-1} f\left(x,\left(1-R^{2}\right)\right)=x .
$$

By the definition of $w(x)$ we have for all $x \in U$ that $w(x)^{\dagger} w(x)=1$ and therefore $R^{2}=x^{\dagger} x=1$ or that

$$
\tilde{f}(x, \hat{0})=\left[\sqrt{\tilde{f}(x, \hat{0})^{\dagger} \tilde{f}(x, \hat{0})}\right] x .
$$

Substitute (29) in the right hand side of (28) to obtain

$$
\begin{aligned}
\frac{\mathrm{d} x}{\mathrm{~d} \tau} & =2\left[\sqrt{\tilde{f}(x, \hat{0})^{\dagger} \tilde{f}(x, \hat{0})}\right]\left[I-x x^{\dagger}\right] x \\
& =2\left[\sqrt{\tilde{f}(x, \hat{0})^{\dagger} \tilde{f}(x, \hat{0})}\right]\left[x-x\left(x^{\dagger} x\right)\right]=\hat{0}
\end{aligned}
$$

and the result follows.

\section{The Explicit Leading Asymptotic Term}

The purpose of this section is to produce conditions that guarantee the existence of solutions $y(t)$ that satisfy 
$\lim _{t \rightarrow t_{\max }^{-}} y(t)=\infty p$ or $\lim _{t \rightarrow t_{\min }^{+}} y(t)=\infty p$ and to determine explicitly the leading asymptotic term in such solutions.

Theorem 14. Let $\infty p$ be a critical point of the polynomial differential system $y^{\prime}=f(y)$ and let $p^{\dagger} f_{L}(p) \neq 0$.

If $p^{\dagger} f_{L}(p)>0$, then the polynomial differential system (17) possesses at least one parameter family of solutions $y(t)$ such that

$$
\begin{aligned}
& y(t) \sim\left\{(L-1) p^{\dagger} f_{L}(p)\left(t_{\max }-t\right)\right\}^{\frac{-1}{L-1}}\{p+o(1)\} \\
& \text { as } t \rightarrow t_{\max }^{-} .
\end{aligned}
$$

If $p^{\dagger} f_{L}(p)<0$, then the polynomial differential system (17) possesses at least one parameter family of solutions $y(t)$ such that

$$
\begin{aligned}
& y(t) \sim\left\{(L-1) p^{\dagger} f_{L}(p)\left(t-t_{\min }\right)\right\}^{\frac{-1}{L-1}}\{p+o(1)\} \\
& \text { as } t \rightarrow t_{\text {min }}^{+} .
\end{aligned}
$$

Proof. We first prove that the differential system (17) is equivalent to

$$
\begin{aligned}
\frac{\mathrm{d}(x-p)}{\mathrm{d} \tau}=A(x-p)+V, \\
A:=2\left(I-p p^{\dagger}\right)\left[J f_{L}(p)-2 f_{L-1}(p) p^{\dagger}\right] \\
\quad-2 p^{\dagger} f_{L}(p) I
\end{aligned}
$$

where $I$ denotes the $k$ by $k$ identity matrix, $J f_{L}(p)$ is the Jacobian matrix of $f_{L}(x)$ evaluated at $x=p$ and $V$ is a polynomial vector function of the vector variables $x, p$ and $(x-p)$ and is such that

$$
V=\mathcal{O}\left(\|x-p\|^{2}\right) \text {, as }\|x-p\| \rightarrow 0
$$

Let $a, b, c$ be three $k$ dimensional column vectors. Then, one can easily verify that the following (non associative and non commutative) relations hold

$$
\left(a^{\dagger} b\right) c=\left(b^{\dagger} a\right) c=\left(c a^{\dagger}\right) b=\left(c b^{\dagger}\right) a .
$$

Notice that

$$
\begin{aligned}
1-R^{2} & =1-\sum_{i=1}^{n}(x-p+p)^{\dagger}(x-p+p) \\
& =-\left[2 p^{\dagger}+(x-p)^{\dagger}\right](x-p)
\end{aligned}
$$

Hence, as $x \rightarrow p$ we have

$$
\begin{aligned}
& \left(1-R^{2}\right)^{L} \\
& =(-1)^{L} \sum_{j=0}^{L} C_{j}^{L}\left[2 p^{\dagger}(x-p)\right]^{j}\left[(x-p)^{\dagger}(x-p)\right]^{L-j} \\
& \leq \mathcal{O}\left[(x-p)^{\dagger}(x-p)\right]^{\frac{L}{2}}=\mathcal{O}\left(\|x-p\|^{L}\right) .
\end{aligned}
$$

$C_{j}^{L}$ are of course the binomial coefficients. We now focus on the expansion of $\tilde{f}$ into a polynomial that depends on the variable $(x-p)$.

Notice that

$$
f_{L}(x)=\left\{f_{L}(p)+\left[J f_{L}(p)\right](x-p)+\Delta_{2}\right\},
$$

where $\Delta_{2}$ is a polynomial of degree 2 in the vector variable $(x-p)$.

We focus on the term $\left(1-R^{2}\right)^{1} f_{L-1}(x)$ in $\tilde{f}$. With the help of (35) we have

$$
\begin{aligned}
\left(1-R^{2}\right)^{1} f_{L-1}(x) & =-\left[2 p^{\dagger}+(x-p)^{\dagger}\right](x-p)\left[f_{L-1}(p)+\left(f_{L-1}(x)-f_{L-1}(p)\right)\right] \\
& =-2 p^{\dagger}(x-p) f_{L-1}(p)+\Delta_{3}=-2 f_{L-1}(p) p^{\dagger}(x-p)+\Delta_{3}
\end{aligned}
$$

where $\Delta_{3}=\mathcal{O}\left(\|x-p\|^{2}\right)$ as $x \rightarrow p$. In sum we have

$$
\begin{aligned}
\tilde{f}\left(x,\left(1-R^{2}\right)\right) & \Delta_{4}:=\Delta_{2}+\Delta_{3}=\mathcal{O}\left(\|x-p\|^{2}\right), \\
=f_{L}(p)+\left[J f_{L}(p)-2 f_{L-1}(p) p^{\dagger}\right](x-p)+\Delta_{4}, & (38) \quad \begin{array}{r}
\text { as } x \rightarrow p \text { and } \Delta_{4} \text { is a polynomial in the variable } \\
(x-p) . \text { Notice that }
\end{array} \\
\left(1+R^{2}\right) I= & {\left[2-\left(1-R^{2}\right)\right] I=\left\{2+\left[2 p^{\dagger}+(x-p)^{\dagger}\right](x-p)\right\} I=2 I+\left[2 p^{\dagger}(x-p)\right] I+\left[(x-p)^{\dagger}(x-p)\right] . } \\
x x^{\dagger}=(x-p+p)\left(x^{\dagger}-p^{\dagger}+p^{\dagger}\right)=p p^{\dagger}+(x-p) p^{\dagger}+p\left(x^{\dagger}-p^{\dagger}\right)+(x-p)\left(x^{\dagger}-p^{\dagger}\right) & \\
\left(1+R^{2}\right) I-2 x x^{\dagger}= & 2 I+\left[2 p^{\dagger}(x-p)\right] I+\left[(x-p)^{\dagger}(x-p)\right] I-2 p p^{\dagger}-2(x-p) p^{\dagger}-2 p\left(x^{\dagger}-p^{\dagger}\right)-2(x-p)\left(x^{\dagger}-p^{\dagger}\right) \\
= & 2\left(I-p p^{\dagger}\right)+\left[2 p^{\dagger}(x-p)\right] I+\left[(x-p)^{\dagger}(x-p)\right] I-2(x-p) p^{\dagger}-2 p\left(x^{\dagger}-p^{\dagger}\right)-2(x-p)\left(x^{\dagger}-p^{\dagger}\right) \\
= & 2\left(I-p p^{\dagger}\right)+\left[2 p^{\dagger}(x-p)\right] I-2(x-p) p^{\dagger}-2 p\left(x^{\dagger}-p^{\dagger}\right)-2(x-p)\left(x^{\dagger}-p^{\dagger}\right)+\mathcal{O}\left(\|x-p\|^{2}\right) .
\end{aligned}
$$

where 
Hence,

$$
\begin{aligned}
\frac{\mathrm{d} x}{\mathrm{~d} \tau}= & \frac{\mathrm{d}(x-p)}{\mathrm{d} \tau}=\left[\left(1+R^{2}\right) I-2 x x^{\dagger}\right] \tilde{f}\left(x,\left(1-R^{2}\right)\right) \\
= & \left\{2\left(I-p p^{\dagger}\right)+\left[2 p^{\dagger}(x-p)\right] I-2(x-p) p^{\dagger}-2 p\left(x^{\dagger}-p^{\dagger}\right)+\mathcal{O}\left(\|x-p\|^{2}\right)\right\} \\
& \times\left\{f_{L}(p)+\left[J f_{L}(p)-2 f_{L-1}(p) p^{\dagger}\right](x-p)+\mathcal{O}\left(\|x-p\|^{2}\right)\right\} \\
= & 2\left(I-p p^{\dagger}\right) f_{L}(0)+\left[2 p^{\dagger}(x-p)\right] f_{L}(p)-2(x-p) p^{\dagger} f_{L}(p)-2 p\left(x^{\dagger}-p^{\dagger}\right) f_{L}(p) \\
& +2\left(I-p p^{\dagger}\right)\left[J f_{L}(p)-2 f_{L-1}(p) p^{\dagger}\right](x-p)+\mathcal{O}\left(\|x-p\|^{2}\right)
\end{aligned}
$$

We analyze the above formula. Observe that by virtue of $\infty p$ being a critical point

$$
\left(I-p p^{\dagger}\right) f_{L}(p)=\left(p^{\dagger} f_{L}(p)\right)\left(p-\left(p p^{\dagger}\right) p\right)=\mathbf{0}
$$

Moreover, by virtue of (34) we have

$$
\begin{aligned}
& {\left[2 p^{\dagger}(x-p)\right] f_{L}(p)-2\left[p\left(x^{\dagger}-p^{\dagger}\right)\right] f_{L}(p) \equiv \mathbf{0}} \\
& {\left[2 p^{\dagger}(x-p)\right] f_{L}(p)-2\left[(x-p) p^{\dagger}\right] f_{L}(p)} \\
& -2\left[p\left(x^{\dagger}-p^{\dagger}\right)\right] f_{L}(p) \equiv-2\left(p^{\dagger} f_{L}(p)\right)(x-p)
\end{aligned}
$$

and the formula (32) follows.

Next we observe that $p^{\dagger}$ is a left eigenvector of $A$.

$$
\begin{aligned}
p^{\dagger} A= & p^{\dagger}\left\{2\left(I-p p^{\dagger}\right)\left[J f_{L}(p)-2 f_{L-1}(p) p^{\dagger}\right]\right\} \\
& -\left[2 p^{\dagger} f_{L}(p)\right] p^{\dagger} I \\
= & -\left[2 p^{\dagger} f_{L}(p)\right] p^{\dagger}
\end{aligned}
$$

because

$$
p^{\dagger}\left\{2\left(I-p p^{\dagger}\right)\right\}=2\left[p^{\dagger}-\left(p^{\dagger} p\right) p^{\dagger}\right]=2\left[p^{\dagger}-p^{\dagger}\right]=\mathbf{0}^{\dagger} .
$$

Thus, $-\left[2 p^{\dagger} f_{L}(p)\right]$ is an eigenvalue of $A$. Assume that $p^{\dagger} f_{L}(p)>0$. Then, it is well known, see Hartman [25] that the differential system (17) possesses at least a one parameter family of solutions such that $\left\|x\left(\tau_{0}\right)-p\right\|$ is small and, such that $x\left(\tau_{0}\right)^{\dagger} x\left(\tau_{0}\right)<1$ and such that $\lim _{\tau \rightarrow \infty} x(\tau)=p$. Furthermore, because of the negative eigenvalue $-\left[2 p^{\dagger} f_{L}(p)\right]$ we have for every fixed $0<\varepsilon<2 p^{\dagger} f_{L}(p)$

$$
\|x(\tau)-p\|=\mathcal{O}\left(\exp \left\{\left[\varepsilon-2 p^{\dagger} f_{L}(p)\right] \tau\right\}\right) \text {, as } \tau \rightarrow \infty
$$

and consequently

$$
\begin{aligned}
& \left\|x^{\dagger} \tilde{f}(x, v)-p^{\dagger} f_{L}(p)\right\|=\mathcal{O}\left(\exp \left\{\left[\varepsilon-2 p^{\dagger} f_{L}(p)\right] \tau\right\}\right), \\
& \text { as } \tau \rightarrow \infty
\end{aligned}
$$

and

$$
\left(1-R^{2}\right)=\mathcal{O}\left(\exp \left\{\left[\varepsilon-2 p^{?} f_{L}(p)\right] \tau\right\}\right), \text { as } \tau \rightarrow \infty .
$$

The formula

$$
t_{\max }-t_{0}=\int_{0}^{\infty}\left(1+R^{2}\right)\left(1-R^{2}\right)^{L-1} \mathrm{~d} \tau
$$

guarantees that $t_{\max }$ is finite because

$$
t_{\text {max }}=t_{0}+\int_{0}^{\infty} \mathcal{O}\left(\exp \left\{\left[\varepsilon-2 p^{\dagger} f_{L}(p)\right] \tau\right\}\right) \mathrm{d} \tau<\infty .
$$

Our next aim is to determine the leading term of $\left(1-R^{2}\right)$ as $t \rightarrow t_{\max }^{-}$. Put $v=\left(1-R^{2}\right)$ in

$$
\begin{aligned}
& \frac{\mathrm{d}\left(1-R^{2}\right)}{\mathrm{d} t}=-\frac{2\left(x^{\dagger} \tilde{f}\left(x,\left(1-R^{2}\right)\right)\right)}{\left(1+R^{2}\right)\left(1-R^{2}\right)^{L-2}}, \\
& \left(1-R^{2}(0)\right)=1-x\left(\tau_{0}\right)^{\dagger} x\left(\tau_{0}\right)>0 .
\end{aligned}
$$

Observe then that the numerator and denominator in

$$
\begin{aligned}
\frac{\mathrm{d} v}{\mathrm{~d} t} & =\frac{-2\left(x^{\dagger} \tilde{f}(x, v)\right)}{(2-v) v^{L-2}} \\
& =\frac{-2\left\{p^{\dagger} f_{L}(p)+\left[x^{\dagger} \tilde{f}(x, v)-p^{\dagger} f_{L}(p)\right]\right\}}{(2-v) v^{L-2}}
\end{aligned}
$$

preserve sign and establish a one to one correspondence among the variables $t, v, \tau$. Notice that

$t \rightarrow t_{\max }^{-} \Leftrightarrow v \rightarrow 0^{+} \Leftrightarrow \tau \rightarrow \infty$. Therefore, there exist sets $\left(\alpha_{0}, t_{\max }^{-}\right),\left(0, v_{0}\right)$ and $\left(\tau_{0}, \infty\right)$ such that for $t \in\left(\alpha_{0}, t_{\max }^{-}\right)$, $v \in\left(0, v_{0}\right)$ and $\tau \in\left(\tau_{0}, \infty\right)$ the integration of (40) yields

$$
\int_{0}^{v}(2-v) v^{L-2} \mathrm{~d} v=\int_{t}^{t_{\max }} 2\left\{p^{\dagger} f_{L}(p)+o(1)\right\} \mathrm{d} t
$$

or

$$
2(L-1)^{-1} v^{L-1}-L^{-1} v^{L}=2\left(t_{\max }-t\right)\left\{p^{\dagger} f_{L}(p)+o(1)\right\} .
$$

As $t \rightarrow t_{\max }^{-}$or $v \rightarrow 0^{+}$or we obtain from the above

$$
\begin{aligned}
& v^{L-1} \sim(L-1)\left(t_{\max }-t\right)\left\{p^{\dagger} f_{L}(p)\right\} \\
& \Leftrightarrow\left(1-R^{2}\right)=v \sim\left\{(L-1) p^{\dagger} f_{L}(p)\left(t_{\max }-t\right)\right\}^{\frac{1}{L-1}} .
\end{aligned}
$$

Thus we obtain as $t \rightarrow t_{\max }^{-}$or $v \rightarrow 0^{+}$or $\tau \rightarrow \infty$ 


$$
\begin{aligned}
y(t) & =\left(1-R^{2}\right)^{-1} x \\
& \sim\left\{(L-1) p^{\dagger} f_{L}(p)\left(t_{\max }-t\right)\right\}^{\frac{-1}{L-1}}\{p+(x-p)\},
\end{aligned}
$$

and the result (30) follows. A similar analysis leads to the desired result if $p^{\dagger} f_{L}(p)<0$.

Remark 15. Our results are an improvement on Elias and Gingold and Gingold and Solomon [21,22] because; 1) we do not assume that all eigenvalues of $A$ have negative real part as in [21], 2) in contrast to [21,22] we express explicitly an eigenvalue of $A$ in terms of the critical direction $p, 3)$ the coefficient of the leading term in the asymptotic formulas (30) and (31) is explicit and does not contain an unknown constant. Theorem 14 shows how the nonlinearity $L$ of our dynamical system and the critical point $\infty p$ determine precisely the asymptotic leading term. We could not find in the following sample of textbooks on differential equations, [4,7-9,23-28], the above explicit asymptotic formulas. Notice that the coefficient matrix A depends on $f_{L-1}(y)$ and not only on the highest degree term $f_{L}(y)$ of the vector field $f(y)$. This is counter intuitive.

Corollary 16. Let $\infty p$ be a critical point of the polynomial differential system $y^{\prime}=f(y)$. Let $p^{\dagger} f_{L}(p) \neq 0$ and let $L>2$. Then the system $y^{\prime}=f(y)$ does not have the Painleve property. Namely, not all of its moving singularities are simple poles.

We turn to the completeness issue.

Definition 17. A differential system $y^{\prime}=f(y)$ is called complete if the solutions to all initial value problems

$$
y^{\prime}=f(y), y\left(t_{0}\right)=w \in \mathbb{R}^{k}
$$

exist on $(-\infty, \infty)$. Otherwise the system is called incomplete.

Studies of "completeness"' questions in nonlinear dynamical systems include $[11,29,30]$ and references therein. The fact that compactification is central to understanding completeness as well as incompleteness is seen from the following theorem. We cannot see how the Gronwall lemma can be used to prove incompleteness.

Theorem 18. Given the polynomial system $y^{\prime}=f(y)$, let $\infty p$ be a critical point such that $p^{\dagger} f_{L}(p) \neq 0$ Then, $y^{\prime}=f(y)$ is incomplete. A necessary condition for a polynomial dynamical system to be complete is that the real part of the eigenvalues of the Jacobians about all critical points $\infty p$ be purely imaginary.

Proof. Use Theorem 14.

Remark 19. It appears that in spite of a voluminous literature on dynamical systems, at least two important outstanding questions remain unresolved. What is the actual interval of existence of their solutions as a function of the initial conditions? For which range of the parameters can we assert that $y^{\prime}=f(y)$ possesses solu- tions that exist on the semi infinite interval $\left(t_{0}, \infty\right)$ ? The analysis of non isolated critical points also needs more illumination.

\section{Observe:}

Remark 20. A leading asymptotic term of singular solutions may be written in the form $V(t)$ where $V(t)$ is an unbounded vector function at a singular point say $t_{0}$. A powerful technique of asymptotic analysis assumes a form $V(t)=S\left(t-t_{0}\right)^{\alpha}$ where the power $\alpha$ is obtained first by so called "balancing". It is only afterwards that a constant vector $S$ is determined. $S$ is to be derived as a solution of a nonlinear system of algebraic equations that could be difficult to solve and is yet to become explicit. This technique of asymptotic analysis was extended refined and applied by various authors. Compare e.g. with [28,31-47]. Applications of this technique to partial differential equations may be found in e.g. Ablowitz and Segur, [31]. This article pursues a different order of operations in the determination of the leading term $V(t)$. Compatification coupled with the identification of equilibrium points of a dynamical system helps first determine the constant vector $S$ from a definite explicit system of nonlinear algebraic equations. Namely,

$$
\mu p=f_{L}(p), \mu=p^{\dagger} f_{L}(p), p^{\dagger} p=1 \text { and } S=\frac{p}{p^{\dagger} f_{L}(p)} \text {. }
$$

\section{An Application to the Competing Species Model}

The competing species model $y^{\prime}=\frac{\mathrm{d} y}{\mathrm{~d} t}=f(y)$ is a polynomial differential system of degree 2 where the vector function $f(y)$ is given by

$$
f(y)=f_{1}(y)+f_{2}(y)
$$

where $f_{j}(y), j=1,2$, are the following homogeneous polynomials column vectors of degree $j$.

$$
\begin{aligned}
& f_{1}(y)=\left[\begin{array}{c}
a y^{1} \\
g y^{2}
\end{array}\right], f_{2}(y)=-\left[\begin{array}{c}
b\left[y^{1}\right]^{2}+c y^{1} y^{2} \\
m y^{1} y^{2}+q\left[y^{2}\right]^{2}
\end{array}\right], \\
& \frac{\mathrm{d} y}{\mathrm{~d} t}=f_{1}(y)+f_{2}(y) .
\end{aligned}
$$

In this section $I$ denotes the 2 by 2 identity matrix.

The competing species model has attracted much attention. Coppel, [48], attests to the large number of quadratic differential systems that model various natural phenomenon, from fluid mechanics to stellar constellations. They share similar features with the competing species model. Compare e.g. with $[7,38,44,48,49]$. For a partial glimpse into the immense literature on quadratic systems see Artes et al., Dumortier et al., Hua et al., Ince, Rein [48-52]. 
The reader should have no difficulty recognizing (43) below as a special case of formula (30) with $L=2$. The theorem below is part of a detailed analysis that can be found in H. Gingold [53]. The theorem reads

Theorem 21. Given (42). Assume that $a, b, c, g, m, q$ $\in \mathbb{R}^{+}$. Then, 1) With one exception all critical points $\infty p$ are generic, namely, $\left.p^{\dagger} f_{2}(p) \neq 0 ; 2\right)$ The basin of divergence of every generic critical point $\infty p$, contains at least a one dimensional manifold; 3) A solution of $y^{\prime}=f(y)$, diverges to $\infty p$ where $\infty p$ is generic if and only if

$$
y(t) \sim \frac{p}{p^{\dagger} f_{2}(p)\left(t_{\max }-t\right)} \text { as } t \rightarrow t_{\max }^{-} .
$$

4) In the exceptional case where $m c-q b=0$, the real valued solutions may exist on the entire real line or may possess singularities with an asymptotic leading term similar to the leading asymptotic term in a Laurent series expansions with a pole of order one or two.

It is interesting to compare the results obtained by Hille, [39,40], where psi series representations for solutions of (42) are obtained for a special range of the parameters. Naturally, these psi series provide explicitly the desired leading asymptotic terms of singular solutions of (42). However, our approach covers numerous cases where the results in $[39,40]$ do not apply.

A detailed analysis of the competing species model of complex valued solutions of $x$ being the independent complex variable, was undertaken by Garnier, [38]. It is not impossible to derive the leading asymptotic terms of singular solutions by the methods presented in [38]. However, this would entails the extraction of the leading asymptotic term of singular solutions of (42) from a myriad of transformations. Another indirect method that could lead to (43) requires the reduction of (42) to a certain pair of second order differential equations satisfied by each component of the vector $y$. The techniques of Bureau, [33,34], may be then applied. It is noteworthy that a more general quadratic system than (42) is not amenable to the results of [34]. This is so because then each component of $y$ could satisfy a second order differential equation $u^{\prime \prime}=h\left(u, u^{\prime}, x\right)$ where $h\left(u, u^{\prime}, x\right)$ is not a rational function of $u$ and $u^{\prime}$. The references mentioned in this paragraph are part of a voluminous literature that deals with an outstanding question that originated with Fuchs, (1884). It stimulated a large amount of work on nonlinear differential equations of the form $u^{\prime \prime}=h\left(u, u^{\prime}, x\right)$, where $h\left(u, u^{\prime}, x\right)$ is a scalar rational function of $u$ and $u^{\prime}$ that possesses coefficients that are analytic functions of the independent variable $x$. The outstanding question is: which equations of the form $u^{\prime \prime}=h\left(u, u^{\prime}, x\right)$ possess solutions that have fixed singularities at certain fixed values of $x$. Thus, mimicking a property of linear non autonomous differential equations.
(These singularities also called by a large school of authors critical points and are not to be confused with the critical points of dynamical systems that are synonymous with equilibrium points of dynamical systems). Other related works include [34-36,43]. A detailed account that lead to the Painleve transcendentals can be found in [26]. Applications to soliton theory may be found in [31]. It is noteworthy that a successful application of the technique in [32] that pursues "closed form" solutions of (42), requires knowledge of the properties of one non constant vector solution of (42).

\section{The Lorenz Completeness, a New Repeller, and an Extension of the Attractor}

In this section we discuss a result whose corollary shows that the Lorenz system is complete for all its real parameters. This completeness property is shared by a larger family of non-autonomous quadratic systems that is denoted below by $\mathcal{N} \mathcal{A} \mathcal{L}$. Then we show that the Lorenz system has a repeller at $\infty$, a corollary of which is the existence of an attractor for the Lorenz system for $\rho \leq 0$.

By a Lorenz system [54] we mean a system satisfying

$$
\begin{aligned}
& \dot{y}_{1}=\sigma\left(y_{2}-y_{1}\right) \\
& \dot{y}_{2}=\rho y_{1}-y_{2}-y_{1} y_{3} \\
& \dot{y}_{3}=-\beta y_{3}+y_{1} y_{2},
\end{aligned}
$$

with $\sigma>0, \beta>0, \rho \in \mathbb{R}$. Note that most authors deal only with Lorenz systems with positive parameters, in which realm there is a global attractor. The existence of an attractor for $\rho \leq 0$ is a corollary of our first result.

Definition 22. Let $C B(\mathbb{R})$ be the family of scalar functions continuous and bounded on $\mathbb{R}$. Let $f_{2}(t, y)$ be a column vector in $\mathbb{R}^{k}$ whose components are quadratic forms: $f_{2}(t, y)_{n}=\left(y^{\dagger} f_{2 n}(t) y\right)$, with each $f_{2 n}(t)$ a lower triangular matrix with entries in $C B(\mathbb{R})$. Let $f_{1}(t, y)=f_{1}(t) y$, where $f_{1}(t)$ is a $k \times k$ matrix with entries in $C B(\mathbb{R})$, and let $f_{0}=f_{0}(t)$ be a column vector in $\mathbb{R}^{k}$ with entries in $C B(\mathbb{R})$. Then $\mathcal{N} \mathcal{A} \mathcal{L}$ (NonAutonomous Lorenz-like) is the class of systems

$$
\dot{y}=f_{2}(t, y)+f_{1}(t, y)+f_{0}(t) \text {, with } y^{\dagger} f_{2}(t, y)=0 \text {. }
$$

The completeness of $\mathcal{N} \mathcal{A L}$ is given in [22], which includes a more detailed description of the structure of $\mathcal{L}$ (autonomous Lorenz-like systems) that could explain the orthogonality property in (45) as a source of the completeness. Obviously, the Lorenz system is in $\mathcal{N} \mathcal{A} \mathcal{L}$ for all real values of its parameters.

Theorem 23. All systems in $\mathcal{N} \mathcal{A} \mathcal{L}$ are complete.

It is shown in [21] that given initial data, the initial value problems (17)-(19) possess unique solutions on $-\infty<\tau<\infty$ such that $\|x(\tau)\| \leq 1$. In particular, it is easy to see from (19) that the boundary sphere $R=1$ is invariant. Thus we may consider the flow on the boundary. 
Setting $R=1$ in (17) reduces it to $x^{\prime}=2 f_{2}=2\left(0,-x_{1} x_{3}, x_{1} x_{2}\right)^{\dagger}$, which is readily solved. There are critical points at $( \pm 1,0,0)^{\dagger}$ and the entire circle $x_{1}=0$. The non-constant solutions are circles in $x_{2}$ and $x_{3}$, with $x_{1}$ fixed:

$$
\hat{x}(\tau)=\left(\begin{array}{c}
a \\
\sqrt{1-a^{2}} \cos (2 a \tau+\delta) \\
\sqrt{1-a^{2}} \sin (2 a \tau+\delta)
\end{array}\right),
$$

where $|a| \leq 1$, and $\delta$ is related to the starting point $\left(a, \sqrt{1-a^{2}} \cos \delta, \sqrt{1-a^{2}} \sin \delta\right)^{\dagger}$. Note that the critical points are limiting cases of the circles as $a \rightarrow \pm 1$ or 0 . For ease of visualization let us orient the axes so that $x_{1}$ "points up". Then the periodic orbits on the unit sphere may be viewed as circles of constant latitude. Note that the period is $\pi / a$ so the motion is very slow near the equator, and the equator full of critical points is a limiting case. If viewed looking down (that is, in along the positive $x_{1}$-axis), orbits in the upper hemisphere rotate counter-clockwise, and those in the lower hemisphere rotate clockwise.

Since $\|\hat{x}(\tau)\|=1, \hat{x}$ does not correspond under the compactification to anything known in the Lorenz system. However, these orbits could be interpreted to correspond to ideal solutions $y(t) \equiv \infty$ that belong to the ultra extended $\mathbb{R}^{3}$. In fact, to consider large $\|y\|$ as $\|y\| \rightarrow \infty$, we restrict our attention to the highest order terms and solve the approximate Lorenz system

$$
\begin{aligned}
& \dot{y}_{1}=0 \\
& \dot{y}_{2}=-y_{1} y_{3} \\
& \dot{y}_{3}=y_{1} y_{2},
\end{aligned}
$$

whose solution is easily seen to be (large) circles in $y_{2}$ and $y_{3}$, with $y_{1}$ constant:

$$
\hat{y}(t)=\left(\begin{array}{c}
C_{1} \\
C_{2} \cos \left(C_{1} t+\delta\right) \\
C_{2} \sin \left(C_{1} t+\delta\right)
\end{array}\right),
$$

where $C_{1}, C_{2}$ and $\delta$ define the starting point $\hat{y}(0)=\left(C_{1}, C_{2} \cos \delta, C_{2} \sin \delta\right)^{\dagger}$. The limits of these circles as $\|y\| \rightarrow \infty$ do not exist in $\mathbb{R}^{3}$, but they can be understood as orbits in the ideal set $I D$, which bounds $\mathbb{R}^{3}$. Let $C_{1}^{2}+C_{2}^{2}$ be large. Then these periodic vector solutions pose a certain enigma. They cannot be interpreted as natural approximations to solutions of the Lorenz system on an infinite time interval, because all solutions must enter a certain ellipsoid in forward time [63]. We choose $C_{1}=r a$ and $C_{2}=r \sqrt{1-a^{2}}$ for $0<|a|<1$. Then as $r \rightarrow \infty, \hat{y}$ transforms under compactification to a circle on the unit sphere with constant first coordi- nate. Choosing instead any finite $C_{1}$ leads to a family of circles, all of which transform to the equator. Similarly, choosing a finite $C_{2}$ leads to a family of circles which transform to the poles.

Definition 24. We say that a surface in $\mathbb{R}^{3}$ is a periodicity surface for the system $\dot{y}=f(y)$ if it is the union of periodic orbits including critical points, and it is the maximal such object in some neighborhood of itself.

The discussion above may be summarized by:

Proposition 25. The ideal set ID is the pre-image of the boundary sphere $\partial U$, which is a periodicity surface of the compactified Lorenz system (17). The periodic orbits are circles that are limit cycles when restricted to any of the planes with $x_{1}$ fixed, $0<\left|x_{1}\right|<1$.

Remark 26. There is great interest in Hilbert's 16th problem asking for the number of limit cycles in planar polynomial differential systems [55-62]. Poincaré is credited with the discovery of limit cycles at infinity of planar polynomial systems $[1,2]$, which are not part of the official count of total limit cycles in the original Hilbert's 16th problem. It is natural now to view the set $I D$ as a periodicity surface of the Lorenz system at infinity and to ask which dynamical systems possess a periodicity surface at infinity.

If the circles on the boundary sphere can be shown to attract nearby orbits (from inside the unit ball) in backwards time $\tau$, it should be possible to say something about asymptotic behavior (in backwards time $t$ ) of the Lorenz equation. This suggests limit cycles at infinity. It is easy enough to show; see e.g., [63], that all trajectories eventually enter a compact set and do not leave it. So it seems plausible that in some sense $\infty$ is a global repeller. On the other hand,

$$
\frac{1}{2} \frac{\mathrm{d}}{\mathrm{d} t}\|y\|^{2}=-\sigma y_{1}^{2}-y_{2}^{2}-\beta y_{3}^{2}+(\sigma+\rho) y_{1} y_{2}
$$

takes both positive and negative values even for large $\|y\|$. Similarly, if the invariant circles on the boundary sphere are to be seen as repelling, we might hope that $R$ decreases along orbits, at least near the boundary sphere. It does not in general since $S$ takes both positive and negative values.

However, we have in [64] proven via a Poincaré map argument on the compactified system

Theorem 27. The ideal set at infinity ID is a global repeller in the following sense: If $r\left(t_{1}\right)=\left\|y\left(t_{1}\right)\right\|$ is large enough, then there exists a $t_{2}>t_{1}$ such that

$r\left(t_{1}\right)>r\left(t_{2}\right)$.

Remark 28. Even though every eigenvalue of the Jacobian at every critical point on the boundary sphere of the compactified Lorenz system has real part equal to zero, we showed that the sphere repels nearby orbits.

Corollary 29. The Lorenz system has an attractor.

Proof. In the proof of the theorem, we established that the boundary sphere repels. Thus the boundary sphere is 
the $\alpha$-limit set of some neighborhood of itself. The $\omega$ limit closure of the complement of that neighborhood is an attractor for the compactified system. Perforce that set is compact, and its uncompactification is the attractor for the Lorenz system, extending the known attractor to the case of $\rho \leq 0$.

Remark 30. Numerous research articles were written on the sensitivity of the Lorenz attractor system. It was labeled as a strange attractor. Its geometrical, analytical topological and probablistic nature has been a subject of numerous investigations. The interested reader may want to consult $[65,66]$ and their references.

\section{Fields of Lorenz-Like Systems near $\infty$}

This section contains results that have not been published elsewhere. A main purpose of our analysis is to show how the behavior of the compactified system on the boundary sphere indicates behavior of solutions and their derivatives of the original system for large $\|y\|$. This is helpful since the compactified system near $R=1$ is usually much simpler than the original system. The asymptotic behavior of the order of growth of the higher derivatives; $\frac{\mathrm{d}^{l} y}{\mathrm{~d} t^{l}}, l=0,1,2, \cdots$ follows, under appropriate conditions, as a bonus from a formula that provides the asymptotic directions of the derivatives.

We first exhibit the relevance of the highest-order system $\dot{y}=f_{2}(y)$ to the original system through analysis of the moving triple $T, N, B$ of unit tangent, normal, and binormal vectors. We can show that $T_{y}, N_{y}, B_{y}$, for trajectories in $\mathcal{L}\left(\mathbb{R}^{3}\right)$ and those of the compactified trajectories $T_{x}, N_{x}, B_{x}$ approach those of $\dot{y}=f_{2}$ as $R \rightarrow 1$. The continuity properties of the compactified differential equation on the compact unit ball then imply that for large $\|y\|, y$ looks a lot like the solutions of $\dot{y}=f_{2}(y)$. In fact, the relevance of the compactified system extends to all orders of derivatives. We stress that we do not expect such correspondence of vector fields for systems not in $\mathcal{L}$. In this section and the next section we denote $y^{\prime}=\frac{\mathrm{d} y}{\mathrm{~d} t}$.

For the strictly second degree polynomial system $\dot{y}=f_{2}(y)$ in $\mathcal{L}\left(\mathbb{R}^{3}\right)$, a direct calculation shows that

$$
\begin{aligned}
& T_{2}=\frac{f_{2}}{\left\|f_{2}\right\|}, \\
& N_{2}=\frac{\left\|f_{2}\right\|^{2}\left[D f_{2}\right] f_{2}-\left(f_{2}^{\dagger}\left[D f_{2}\right] f_{2}\right) f_{2}}{\|\| f_{2}\left\|^{2}\left[D f_{2}\right] f_{2}-\left(f_{2}^{\dagger}\left[D f_{2}\right] f_{2}\right) f_{2}\right\|}, \\
& B_{2}=\frac{f_{2} \times\left(\left[D f_{2}\right] f_{2}\right)}{\left\|f_{2} \times\left(\left[D f_{2}\right] f_{2}\right)\right\|},
\end{aligned}
$$

where we use the notation $\left[D f_{i}\right]$ for the Jacobian of the vector function $f_{i}$. The expressions in (49) are given as functions of the variable $y$; however, it is easy to see that the values of the expressions are not changed if they are expressed in terms of $x$. Unless otherwise indicated, all subsequent occurrences of $f_{i}$ and $\left[D f_{i}\right]$ are to be understood as $f_{i}(x)$ and $\left[D f_{i}(x)\right]$.

Theorem 31. Let $y \in \mathcal{L}\left(\mathbb{R}^{3}\right)$. Then the triples $T_{y}, N_{y}$, $B_{y}$ and $T_{x}, N_{x}, B_{x}$ coincide with $T_{2}, N_{2}, B_{2}$, plus terms of order $O\left(\frac{1-R^{2}}{\left\|f_{2}\right\|}\right)$ as $R^{2} \rightarrow 1$.

Proof. The calculation required to show that both $T_{y}$ and $T_{x}$ approach $T_{2}$, and both $N_{y}$ and $N_{x}$ approach $N_{2}$ as $\left(1-R^{2}\right) \rightarrow 0$ is omitted. Assuming it, and since $B=T \times$ $N$, we also have that $B_{y}$ and $B_{x}$ approach $B_{2}$.

We develop an interesting relationship between $t$-derivatives of $y$ for large $\|y\|$ and $\tau$-derivatives of $x$ near the boundary sphere, but away from critical points and zeros of higher derivatives. We show that for systems in $\mathcal{L}$, the vector fields of higher derivatives of $y$ with respect to $t$ have the same direction as the corresponding derivatives of $x$ with respect to $\tau$ for $\|y\|$ large enough. We stress that this property need not hold for general quadratic systems.

Proposition 32. Let $y \in \mathcal{L}$. Then, for all integers $n \geq 0$, as $\|y\| \rightarrow \infty$ or $R^{2} \rightarrow 1^{-}$, we have

$\left(1-R^{2}\right)^{n+1}\left(1+R^{2}\right)^{n} \mathrm{~d}^{n} y / \mathrm{d} t^{n}=\mathrm{d}^{n} x / \mathrm{d} \tau^{n}+\mathcal{O}\left(1-R^{2}\right)$.

Moreover, the order of growth of the derivatives $\mathrm{d}^{n} y / \mathrm{d} t^{n}$ is given by

$$
\left\|\mathrm{d}^{n} y / \mathrm{d} t^{n}\right\| \leq M_{n}\|y\|^{n+1}, n=0,1,2, \cdots,
$$

where $M_{n}$ are certain constants. Furthermore, for each $n$, let $S_{n}$ be the set of points in the boundary where $\mathrm{d}^{n} x / \mathrm{d} \tau^{n}=0$. Then $\forall \epsilon>0$, there is a neighborhood $U_{n}$ of $S_{n}$ in the closed unit ball, with $m\left(U_{n}\right)<\epsilon,\left(m\left(U_{n}\right)\right.$ being the measure of $U_{n}$ ), such that for $x \in$ the complement of $U_{n}$ and $\|y\| \rightarrow \infty$ or $R^{2} \rightarrow 1^{-}$we have

$$
\frac{\mathrm{d}^{n} y / \mathrm{d} t^{n}}{\left\|\mathrm{~d}^{n} y / \mathrm{d} t^{n}\right\|}=\frac{\mathrm{d}^{n} x / \mathrm{d} \tau^{n}}{\left\|\mathrm{~d}^{n} x / \mathrm{d} \tau^{n}\right\|}+\mathcal{O}\left(1-R^{2}\right)
$$

Proof. (50) can be shown by induction. The conclusions in (51) and (52) follow from (50). The key to the induction is the useful result that

$$
\frac{\mathrm{d}}{\mathrm{d} \tau}\left(1-R^{2}\right)^{n}=2 n S\left(1-R^{2}\right)^{n+1} .
$$

From that, we can prove two interesting formulas: First, for $n \geq 0$, we have

$$
\left(1-R^{2}\right)^{n}\left(1+R^{2}\right)^{n} \frac{\mathrm{d}^{n} x}{\mathrm{~d} t^{n}}=\frac{\mathrm{d}^{n} x}{\mathrm{~d} \tau^{n}}+\left(1-R^{2}\right) D_{n-1} x,
$$


where $D_{n}$ is an $n^{\text {th }}$ degree polynomial in $\frac{\mathrm{d}}{\mathrm{d} \tau}$, with rational (in $x$ ) continuous coefficients in the unit ball. Second, for $n \geq 1$,

$$
\left(1-R^{2}\right)^{n-2}\left(1+R^{2}\right)^{2 n-1} \frac{\mathrm{d}^{n}\left(1-R^{2}\right)}{\mathrm{d} t^{n}}=P_{n}(x),
$$

where $P_{n}(x)$ is a polynomial in $x$.

The reason that Lorenz-like systems distinguish themselves from other nonlinear systems so that the vector fields $\frac{\mathrm{d}^{l} x}{\mathrm{~d} \tau^{l}}$ and $\frac{\mathrm{d}^{l} y}{\mathrm{~d} t^{l}}, l=0,1,2, \cdots$ are asymptotically parallel for large $\|y\|$ can be traced back to the relation (53) which again is a result of the orthogonality in (45). Recall that orthogonality featured also in the completeness result. It basically says that in a Lorenz-Like system $y^{\dagger} f(y)$ does not grow faster than $\|y\|^{2}$ as $\|y\| \rightarrow \infty$.

We now specialize Theorem 31 to the Lorenz system. For the Lorenz system or any system with $x^{\prime}$ proportional to $\left(0,-x_{3}, x_{2}\right)^{\dagger}$, it is easy to see that

$$
\begin{aligned}
& T_{\hat{x}}=\frac{1}{\sqrt{x_{2}^{2}+x_{3}^{2}}}\left(\begin{array}{c}
0 \\
-x_{3} \\
x_{2}
\end{array}\right), \\
& N_{\hat{x}}=\frac{1}{\sqrt{x_{2}^{2}+x_{3}^{2}}}\left(\begin{array}{c}
0 \\
-x_{2} \\
-x_{3}
\end{array}\right), B_{\hat{x}}=\left(\begin{array}{l}
1 \\
0 \\
0
\end{array}\right) .
\end{aligned}
$$

Specializing Theorem 31 to Lorenz systems, the triple $T, N, B$ for an orbit of the Lorenz system and of its compactified version approach those of (56) as $R^{2} \rightarrow 1$ :

Corollary 33. The moving triple T, $N, B$ for the Lorenz system and the compactified version coincide with those of the circle $\hat{x}$, plus terms of order $O\left(\frac{\left(1-R^{2}\right)}{\| x_{1} \mid \sqrt{x_{2}^{2}+x_{3}^{2}}}\right)$ as $R^{2} \rightarrow 1$.

\section{REFERENCES}

[1] H. Poincare, "Mémoire sur les Courbes Définies par Une Équation Différentielle,” Journal de Mathématiques Pures et Appliquées, Vol. 7, 1881, pp. 375-422.

[2] R. K. W. Roeder, “On Poincaré's Fourth and Fifth Examples of Limit Cycles at Infinity,” Rocky Mountain Journal of Mathematics, Vol. 33, No. 3, 2003, pp. 1057-1082. doi:10.1216/rmjm/1181069943

[3] I. Bendixson, "Sur les Courbes Définies par des Équations Différentielles,” Acta Mathematica, Vol. 24, No. 1, 1901, pp. 1-88. doi:10.1007/BF02403068

[4] A. A. Andronov, E. A. Leontovich, I. I. Gordon and A. L. Maier, "Qualitative Theory of Second-Order Dynamic
Systems,” Wiley, New York, 1973.

[5] L. A. Ahlfors, "Complex Analysis," McGraw-Hill, New York, 1979.

[6] E. Hille, “Analytic Function Theory,” Chelsea Publishing Company, New York, 1982.

[7] D. Jordan and P. Smith, "Nonlinear Ordinary Differential Equations: An Introduction for Scientists and Engineers," 4th Edition, In: Oxford Texts in Applied and Engineering Mathematics, 2007.

[8] S. Lefschetz, "Differential Equations: Geometric Theory,” Dover, New York, 1977.

[9] L. Perko, "Differential Equations and Dynamical Systems,” 3rd Edition, In: Texts in Applied Mathematics, Vol. 7, Springer-Verlag, Berlin, 2001.

[10] G. Sansone and R. Conti, "Non-Linear Differential Equations,” Pergamon Press, Oxford, 1964.

[11] C. Chicone and J. Sotomayor, "On a Class of Complete Polynomial Vector Fields in the Plane,” Journal of Differential Equations, Vol. 61, No. 3, 1986, pp. 398-418. doi:10.1016/0022-0396(86)90113-0

[12] A. Cima and J. Llibre, "Bounded Polynomial Vector Fields Bounded Polynomial Vector Fields," Transactions of the American Mathematical Society, Vol. 318, No. 2, 1990, pp. 557-579. doi:10.1090/S0002-9947-1990-0998352-5

[13] D. Schlomiuk and N. Vulpe, "The Full Study of Planar Quadratic Differential Systems Possessing a Line of Singularities at Infinity,” Journal of Dynamics and Differential Equations, Vol. 20, No. 4, 2008, pp. 737-775. doi:10.1007/s10884-008-9117-2

[14] Y. I. Gingold and H. Gingold, "Geometric Properties of a Family of Compactifications,” Balkan Journal of Geometry and Its Applications, Vol. 12, No. 1, 2007, pp. 44-55.

[15] G. Q. Chen and Z. J. Liang, “Affine Classification for the Quadratic Vector Fields without the Critical Points at Infinity," Journal of Mathematical Analysis and Applications, Vol. 172, No. 1, 1993, pp. 62-72. doi:10.1006/jmaa.1993.1007

[16] H. Gingold, "Compactification Applied to a Discrete Competing System Model,” International Journal of Pure and Applied Mathematics, Vol. 66, No. 3, 2011, pp. 297 320.

[17] H. Gingold, "Divergence of Solutions of Polynomials Finite Difference Equations," Proceedings A of the Royal Society of Edinburgh, Vol. 142, No. 4, 2012, pp. 787-804. doi:10.1017/S0308210510000077

[18] H. Gingold, "Compactification and Divergence of Solutions of Polynomial Finite Difference Systems of Equations," Dynamics of Continuous, Discrete and Impulsive Systems Series B: Applied Algorithms, Vol. 18, No. 3, 2011, pp. 315-335.

[19] H. Gingold, “Approximation of Unbounded Functions via Compactification," Journal of Approximation Theory, Vol. 131, No. 2, 2004, pp. 284-305. doi:10.1016/j.jat.2004.08.001

[20] S. Willard, “General Topology,” Addison-Wesley, Reading, 1970. 
[21] U. Elias and H. Gingold, “Critical Points at Infinity and Blow Up of Solutions of Autonomous Polynomial Differential Systems via Compactification,” Journal of Mathematical Analysis and Applications, Vol. 318, No. 1, 2006, pp. 305-322.

[22] H. Gingold and D. Solomon, "On Completeness of Quadratic Systems,” Nonlinear Analysis, Vol. 74, No. 12, 2011, pp. 4234-4240.

[23] J. Guckenheimer and P. Holmes, "Nonlinear Oscillations, Dynamical Systems and Bifurcations of Vector Fields," Springer-Verlag, New York, 1983.

[24] W. Hahn, "Stability of Motion,” Springer Verlag, Berlin, 1967.

[25] P. Hartman, “Ordinary Differential Equations,” 2nd Edition, Birkhauser, 1982.

[26] E. L. Ince, “Ordinary Differential Equations," Dover, New York, 1956.

[27] A. C. J. Luo, "Global Transversality, Resonance, and Chaotic Dynamics,” World Scientific Publishing Co. Pte. Ltd., Hackensack, 2008.

[28] M. Tabor, "Chaos and Integrability in Nonlinear Dynamics,” John Wiley \& Sons, New York, 1989.

[29] B. Coomes, "The Lorenz System Does Not Have a Polynomial Flow,” Journal of Differential Equations, Vol. 82, No. 2, 1989, pp. 386-407. doi:10.1016/0022-0396(89)90140-X

[30] G. Meisters, “Polynomial Flows on $\mathbb{R}^{n}$," Proceedings of the Semester on Dynamical Systems, Mokotowska, 1986, p. 25.

[31] M. J. Ablowitz and H. Segur, "Solitons and the Inverse Scattering Transform,” Society for Industrial and Applied Mathematics, Philadelphia, 1981. doi:10.1137/1.9781611970883

[32] T. C. Bountis, V. Papageorgiou and P. Winternitz, “On the Integrability of Systems of Nonlinear Ordinary Differential Equations with Superposition Principles,” Journal of Mathematical Physics, Vol. 27, No. 5, 1986, pp. 1215-1224. doi:10.1063/1.527128

[33] F. J. Bureau, "Équations Différentielles du Second Ordre en Y et du Second Degré en Ÿ dont l’Intégrale Générale Est á Points Critiques Fixes,” Annali di Matematica Pura ed Applicata, Vol. 91, No. 4, 1972, pp. 163-281.

[34] F. J. Bureau, "Differential Equations with Fixed Critical Points,” Annali di Matematica Pura ed Applicata, Vol. 66, No. 1, 1964, pp. 1-116. doi:10.1007/BF02412437

[35] J. Chazy, "Sur les Équations Différentielles du Troisième Ordre et d'Ordre Supérieur dont l'Intégrale Générale a Ses Points Critiques Fixes,” Acta Mathematica, Vol. 34, No. 1, 1911, pp. 317-385.

[36] J. Chazy, "Sur les Équations Différentielles dont l'Intégrale Générale Possede Un Coupure Essentielle Mobile,” Les Comptes Rendus de l'Académie des Sciences (Paris), Vol. 150, 1910, pp. 456-458.

[37] B. Gambier, “Sur les Équations Différentielles du Second Ordre et du Premier Degré dont l’Intégrale Générale Est a Points Critiques Fixes,” Acta Mathematica, Vol. 33, No. 1, 1910, pp. 1-55. doi:10.1007/BF02393211
[38] R. Garnier, "Sur des Systémes Différentiels du Second Ordre dont l'Intégrale Générale est Uniforme,” Annales Scientifiques de l'École Normale Supérieure, Vol. 77, 1960, pp. 123-144.

[39] E. Hille, "On a Class of Series Expansions in the Theory of Emden's Equation,” Proceedings of the Royal Society of Edinburgh, Section A, Vol. 71, Part 2, 1973, pp. 95110.

[40] E. Hille, “A Note on Quadratic Systems,” Proceedings of the Royal Society of Edinburgh, Section A, Vol. 72, No. 1, 1974, pp. 17-37.

[41] P.-F. Hsieh and Y. Sibuya, "Basic Theory of Ordinary Differential Equations,” Universitext, Springer-Verlag, New York, 1999.

[42] D. D. Hua, L. Cairó, M. R. Feix, K. S. Govinder and P. G. L. Leach, "Connection between the Existence of First Integrals and the Painlevé Property in Lotka-Volterra and Quadratic Systems,” Proceedings of the Royal Society A, Vol. 452, No. 1947, 1996, pp. 859-880.

doi:10.1098/rspa.1996.0043

[43] S. Kowalevski, "Sur le Probléme de la Rotation d’Un Corps Solide Autour d’Un Point Fixe,” Acta Mathematica, Vol. 12, No. 1, 1889, pp. 177-232. doi:10.1007/BF02592182

[44] P. G. L. Leach and J. Miritzis, "Competing Species: Integrability and Stability,” Journal of Nonlinear Mathematical Physics, Vol. 11, No. 1, 2004, pp. 123-133. doi:10.2991/jnmp.2004.11.1.9

[45] P. G. L. Leach, G. P. Flessas and S. Cotsakis, "Symmetry, Singularities and Integrability in Complex Dynamics I: The Reduction Problem," Journal of Nonlinear Mathematical Physics, Vol. 7, No. 4, 2000, pp. 445-479. doi:10.2991/jnmp.2000.7.4.4

[46] M. Tabor and J. Weiss, "Analytic Structure of the Lorenz System,” Physical Review A, Vol. 24, No. 4, 1981, pp. 2157-2167. doi:10.1103/PhysRevA.24.2157

[47] A. Ramani, B. Grammaticos and T. Bountis, "The Painlevé Property and Singularity Analysis of Integrable and Nonintegrable Systems,” Physical Report, Vol. 180, No. 3, 1989, pp. 159-245.

[48] W. A. Coppel, “A Survey of Quadratic Systems,” Journal of Differential Equations, Vol. 2, No. 3, 1966, pp. 293304. doi:10.1016/0022-0396(66)90070-2

[49] S.-B. Hsu, S. Hubbell and P. Waltman, "A Contribution to the Theory of Competing Predators,” Ecological Monographs, Vol. 48, No. 3, 1978, pp. 337-349. doi:10.2307/2937235

[50] J. C. Artes, J. Llibre, N. Vulpe, "Singular Points of Quadratic Systems: A Complete Classification in the Coefficient Space," International Journal of Bifurcation and Chaos, Vol. 18, No. 2, 2008, pp. 313-362. doi:10.1142/S021812740802032X

[51] J. C. Artes, R. E. Kooij and J. Llibre, "Structurally Stable Quadratic Vector Fields,” Vol. 134, Memoirs of the American Mathematical Society, 1998.

[52] F. Dumortier, J. Llibre and J. Artes, "Qualitative Theory of Planar Differential Systems," Universitext, SpringerVerlag, Berlin, 2006. 
[53] H. Gingold, "The Leading Asymptotic Term of Real Valued Solutions of a Competing Species Model,” pp. 1-23.

[54] E. N. Lorenz, "Deterministic Nonperiodic Flow," Journal of the Atmospheric Sciences, Vol. 20, No. 2, 1963, pp. 130-141. doi:10.1175/1520-0469(1963)020<0130:DNF>2.0.CO;2

[55] X.-Y. Chen, “On Generalized Rotated Vector Fields,” Journal of Nanjing University of Posts and Telecommunications (Natural Science), Vol. 1, 1975, pp. 100-108.

[56] G. F. D. Duff, "Limit-Cycles and Rotated Vector Fields," Annals of Mathematics, Vol. 57, No. 1, 1953, pp. 15-31. doi:10.2307/1969724

[57] L. Perko, "Rotated Vector Fields and the Global Behavior of Limit Cycles for a Class of Quadratic Systems in the Plane,” Journal of Differential Equations, Vol. 18, No. 1, 1975, pp. 63-86. doi:10.1016/0022-0396(75)90081-9

[58] A. Yu. Fishkin, "On the Number of Limit Cycles of Planar Quadratic Vector Fields,” Doklady Akademii Nauk (Russian), Vol. 428, No. 4, 2009, pp. 462-464.

[59] J. C. Artés, J. Llibre and J. C. Medrado, "Nonexistence of Limit Cycles for a Class of Structurally Stable Quadratic Vector Fields,” Discrete and Continuous Dynamical Systems, Vol. 17, No. 2, 2007, pp. 259-271.
[60] N. Bautin, “On Periodic Solutions of a System of Differential Equations,” Prikladnaya Matematika i Mekhanika, Vol. 18, 1954, p. 128.

[61] Yu. S. Ilýashenko, "Finiteness Theorems for Limit Cycles," Translated from the Russian by H. H. McFaden. Translations of Mathematical Monographs, 94, American Mathematical Society, Providence, 1991

[62] Y. Yanqian, “Theory of Limit Cycles,” American Mathematical Society, Providence, 1986.

[63] C. Sparrow, "The Lorenz Equations: Bifurcations, Chaos, and Strange Attractors," Vol. 41, Applied Mathematical Sciences, Springer-Verlag, Berlin, 1982. doi:10.1007/978-1-4612-5767-7

[64] H. Gingold and D. Solomon, "The Lorenz System Has a Global Repeller at Infinity,” Journal of Nonlinear Mathematical Physics, Vol. 18, No. 2, 2011, pp. 183-189.

[65] M. Holland and I. Melbourne, "Central Limit Theorems and Invariance Principles for Lorenz Attractors,” Journal of the London Mathematical Society, Vol. 76, No. 2, 2007, pp. 345-364.

[66] B. Marlin, “An Upper Semi-Continuous Model for the Lorenz Attractor,” Topology Proceedings, Vol. 40, 2012, pp. 73-81. 